\title{
New Concepts for Shipboard Sea State Estimation
}

\author{
Nielsen, Ulrik D.; Bjerregård, Mikkel; Galeazzi, Roberto; Fossen, Thor I.
}

Published in:

Proceedings of OCEANS '15

Publication date:

2015

Document Version

Publisher's PDF, also known as Version of record

Link back to DTU Orbit

Citation (APA):

Nielsen, U. D., Bjerregård, M., Galeazzi, R., \& Fossen, T. I. (2015). New Concepts for Shipboard Sea State Estimation. In Proceedings of OCEANS '15 [7404386] IEEE.

\section{General rights}

Copyright and moral rights for the publications made accessible in the public portal are retained by the authors and/or other copyright owners and it is a condition of accessing publications that users recognise and abide by the legal requirements associated with these rights.

- Users may download and print one copy of any publication from the public portal for the purpose of private study or research.

- You may not further distribute the material or use it for any profit-making activity or commercial gain

- You may freely distribute the URL identifying the publication in the public portal

If you believe that this document breaches copyright please contact us providing details, and we will remove access to the work immediately and investigate your claim. 


\title{
New Concepts for Shipboard Sea State Estimation
}

\author{
Ulrik D. Nielsen ${ }^{1,4}$, Mikkel Bjerregård ${ }^{2}$, Roberto Galeazzi ${ }^{2}$ and Thor I. Fossen ${ }^{3,4}$
}

\begin{abstract}
The wave buoy analogy is a tested means for shipboard sea state estimation. Basically, the estimation principle resembles that of a traditional wave rider buoy which relies, fundamentally, on transfer functions used to relate measured waveinduced responses and the unknown wave excitation. This paper addresses however a newly developed concept of the wave buoy analogy but the approach presented herein is, on the contrary, not relying exclusively on transfer functions. Instead, the method combines a signal-based part, estimating wave frequency, and a model-based part, estimating wave amplitude and phase, where only the model-based part depends on transfer functions whereas the signal-based part relies on the measured vessel response alone. Case studies in terms of hypothetical examples show that the method is capable to reconstruct fully the wave elevation process of a sinusoidal regular wave; which include estimation of the wave's frequency, amplitude and phase. At this stage, the method is far from being a useful means in practical, realsituation applications but the method provides, indeed, a valuable step towards developing new approaches for shipboard sea state estimation.
\end{abstract}

\section{INTRODUCTION}

\section{A. Context: Safety and Efficiency of Marine Operations}

For ship masters of marine crafts at sea it is profitable to have available knowledge of the prevailing sea state. In one way or the other, ship masters therefore make or gain estimates of the on-site sea state, as information about it can be used to improve both safety and efficiency of any marine operation, including, e.g., ships in transit, oil and gas production from floating structures, general ship-to-ship actions, installation and maintenance of fixed or floating offshore structures such as wind farms. A concrete example relates to large container and passenger ships, which typically operate with quite narrow operational windows and, at occasions, experience large wave-induced motions and associated responses. However, the increase in vessel size means that it has become ever more difficult for the crew to make precise observations of the seaway by sight, since the relative wave size and severity become increasingly difficult to assess on larger vessels. This is further complicated in rough seas, where quick and accurate decisions have to be made to keep passengers, cargo and equipment safe. It is therefore desirable to develop on-board, real-time decision support systems (DSS), [1]-[7], which will assist the crew to make proper decisions. Thus, the DSS could raise a warning if the vessel were in an operational region where any potentially dangerous phenomenon is likely

\footnotetext{
${ }^{1}$ DTU Mechanical Engineering, Technical University of Denmark, Kgs. Lyngby, Denmark.

${ }^{2}$ DTU Electrical Engineering, Technical University of Denmark.

${ }^{2}$ Department of Engineering Cybernetics, Norwegian University of Science and Technology (NTNU), Trondheim, Norway.

${ }^{4}$ Centre for Autonomous Marine Operations (AMOS), NTNU, Trondheim, Norway.
}

to occur. In less critical situations, a DSS can be used for route planning with respect to fuel consumption so to evaluate and improve vessel and fleet propulsion performance [8], [9]. Other applications include decision support and/or control of dynamic positioning systems, where both stationkeeping behaviour and fuel consumption can be improved by introducing feed-forward control [10], [11].

In general, decision support systems on marine crafts rely on mathematical models relating the particular vessel responses of interest and the on-site sea state. Thus, the on-site sea state is fundamental input to any DSS, as it is directly responsible for the wave-induced loads to be likely experienced by the marine craft in consideration. Obviously, the DSS should be reliable (and accurate), as far as possible, under all conditions and, hence, the sea state estimate should not be based on observations by the ship master but on, objective, measurements. Indeed, the present paper focuses on one such means to estimate a sea state by use of measurements of vessel responses. Specifically, the study provides a review, or a condensed summary, on new approaches developed - and to be developed - to estimate the sea state on operating marine crafts from on-board measurements of wave-induced responses. It is noteworthy that the particular study is much of a conceptual kind, as its practical application very much depends on how successful future work and developments are to become; if properly addressed.

\section{B. Composition of Paper}

The paper is organised into five sections, including the introduction, numbered I-V. Section II gives an overview of methods and approaches for shipboard sea state estimation and the section introduces the inherent problem(s) associated to the means. In Section III, the theoretical aspects of the new estimation concept are outlined, and examples including associated results and discussions of it appear in Section IV. Finally, conclusions are drawn in Section V which also suggests further studies and/or developments.

\section{SHIPBOARD SEA STATE ESTIMATION}

\section{A. Wave Buoy Analogy}

Different means exist to estimate a sea state; often given in terms of characteristic sea state parameters such as significant wave height, mean wave period and mean wave direction. Since the 1970s (moored) directional buoys have been considered as the most reliable and accurate means for sea state estimation. However, traditional wave-rider buoys suffer from being subject to damage and/or loss; and, more importantly, an enormous network would be required to cover all parts of the oceans. For marine operations, considering ships or other floating structures, a more practical and appealing approach is 
to use on-board sensors, where wave-induced responses from the vessel itself provide the basis for an estimate of the on-site sea state [12]-[17]. Until recently, this approach - denoted the wave buoy analogy - has been only explored in a model-based framework and in the frequency domain, where knowledge of transfer function(s) of the vessel is essential. Thus, the governing equation system, having the directional wave spectrum as its solution, relies on spectral analysis where linearity is assumed between waves and the associated wave-induced (measured) response(s); related through the transfer functions. As the outcome consists of the complete energy distribution of the wave system, with frequency and directional information, the approach is applicable to general decision support systems, and, typically, reasonable estimates of the wave spectrum can be expected [18]-[21]. The accuracy of the estimates depends inherently on availability of accurate transfer functions and, moreover, the reliability is highly dependent on the spectral (response) analysis, by for instance fast Fourier transform (FFT) procedures, in which aspects of stationarity influence the outcome [22], [23]. In principle, stationary operational conditions are necessary because a minimum time window, in the order 10-15 minutes, is needed to perform the spectral analysis. The reason is that if conditions are not stationary during the considered period, either because of changing sea state or, more likely, as a result of speed and/or heading changes of the vessel - the sea state estimates are likely to be unreliable. Moreover, the need for a certain minimum time period has another consequence, as it implies that estimates, strictly speaking, will be backdated; which in turn will be of (negative) importance if response predictions are to be made ahead of any measurements [7].

\section{B. Model-based and Signal-based Estimation}

The disadvantages, in terms of a model-based methodology and the requirement related to stationarity, lead to a wish for a purely signal-based version of the wave buoy analogy, not dependent on transfer functions but on measurements solely, and, in combination, a wish for a methodology that efficiently can handle nonstationary conditions. Although it seems like a very difficult task to find approaches accommodating these wishes, in particular the former, work has been initiated in the particular direction and thus signal-based procedures have been developed [24]-[26] to estimate the encountered wave peak frequency $\omega_{p}$, allowing for nonstationary conditions.

The estimation of the peak wave frequency itself is valuable in many applications and, notably, it is useful for ship autopilot and dynamic positioning systems of marine crafts. However, so far a signal-based method has not been developed to estimate additional characteristic wave parameters, which will be necessary towards a complete description of the sea state and, thus, required for general decision support systems for safe and efficient marine operations. An intermediate step in the direction would be a combined signal- and model-based procedure which can handle measurements data that can be nonstationary.

Methodologies of such a combined procedure have recently been addressed [27] and in the following that work and associated theoretical aspects and key elements are reviewed. As pointed out previously, the work still needs elaboration, and focus is restricted to estimation of the fundamental parameters describing a regular wave; that is, frequency, amplitude, and phase. Although this set of parameters allows the sea surface elevation to be fully reconstructed, the practical application to the method's current development is rather limited, which is obviously reflected by the somewhat theoretical examples to be shown later.

\section{THEORY}

\section{A. Conditions and Outcome}

The procedure consists of a signal-based part and a modelbased part, in combination, where the former introduces a filter [28] to identify the wave frequency while the latter part, subsequently, considers the hydrodynamic behaviour of the particular vessel when exposed to a (sinusoidal) seaway. Physically, the model-based part sets up a mathematical model which compares the vessel's theoretical wave-induced behaviour with the corresponding measured behaviour, so that wave amplitude and phase can be estimated.

Without loss of generality the motion response considered in the study is the heave component and, for matters of convenience, the associated response amplitude operator (RAO) is obtained by closed-form expressions. Thus, RAOs have been implemented for a semisubmersible [29] and for a large container ship [30] to produce two different case studies. The case studies deal with numerical simulations (of measurements data) only and, hence, the (absolute and relative) accuracy of the RAOs are of minor importance for what reason nothing further is said about the RAOs.

\section{B. Peak-frequency Estimate}

Purely signal-based methods for estimation of the (encountered) peak frequency of a wave system already exist and a novel procedure [25] has been applied successfully to full-scale response data of an in-service container vessel. In the somewhat conceptual and theoretical study made in the present paper, robustness is not important as it is for realworld applications studied by [25]. Thus, a simpler version of the procedure is considered herein and, consequently, the present method handles, at this stage, only sinusoidal signals. The simplifications could quite easily be relaxed, but as the subsequent estimation of wave amplitude and phase by the model-based part of the present method is restricted (so far) to sinusoidal signals there is no need for a 'global exponential stable nonlinear wave encounter frequency estimator' which cannot estimate amplitude and/or phase.

The filter utilised in this work to estimate in real-time the frequency of a sinusoidal signal was initially derived in [28]. However, herein a slightly more general version of the filter is applied and the outline is given by [27], but with the main points repeated below: 
A sine wave with unknown constant amplitude $A_{y}$, frequency $\omega_{e}$ and phase $\varepsilon$ is given

$$
y(t)=A_{y} \sin \left(\omega_{e} t+\varepsilon\right)
$$

and the objective is to estimate the frequency $\omega_{e}$ on the basis of only noisy measurements of $y(t)$.

Basically, any sinusoidal signal represents the solution to the problem of an undamped harmonic oscillator

$$
\ddot{y}=-\omega_{e}^{2} y=\varphi y
$$

and thus $\varphi=-\omega_{e}^{2}$ is the parameter to be estimated. The equivalent critically-damped mass-spring system with forcing was studied in [28], wherein it was shown that the auxiliary filter:

$$
\begin{aligned}
& \dot{\xi}_{1}=\xi_{2} \\
& \dot{\xi}_{2}=-2 \xi_{2}-\xi_{1}+y
\end{aligned}
$$

with the equivalent second order transfer function

$$
\xi_{1}(s)=\frac{1}{(s+1)^{2}} y(s)
$$

tracks the measured sinusoid; until a cut-off frequency at 1 $\mathrm{rad} / \mathrm{s}$. For any wave with higher frequency the filter can be modified as follows

$$
\xi_{1}(s)=\frac{\omega_{f}^{2}}{\left(s+\omega_{f}\right)^{2}} y(s)
$$

where the cut-off frequency $\omega_{f}$ should be chosen such that $\omega_{f}>\omega_{e}$ to ensure that the auxiliary filter is sufficiently fast to keep track of the wave. The frequency estimator thus becomes, cf. [27]:

$$
\begin{aligned}
\dot{\xi}_{1} & =\xi_{2} \\
\dot{\xi}_{2} & =-2 \omega_{f} \xi_{2}-\omega_{f}^{2} \xi_{1}+\omega_{f}^{2} y \\
\dot{\hat{\varphi}} & =k_{a} \xi_{1}\left(\dot{\xi}_{2}-\hat{\varphi} \xi_{1}\right) \\
\hat{\omega}_{e} & =\sqrt{|\hat{\varphi}|}
\end{aligned}
$$

which often will be referred to as the 'Aranovskiy filter' in the remaining parts of the paper. The original work is given by [28], but it is noteworthy that additional experimental results are found in [25], which also includes a stability proof for global exponential stability.

\section{Wave-amplitude and -phase Estimates}

C.1 Nonlinear least squares fitting:

Application of the Aranovskiy filter on any (sinusoidal) signal facilitates determination of the (peak) frequency of the signal. Thus, the filter can be directly applied to real-time vessel response measurements and the task left is to make estimates of the wave amplitude and phase. In case of a modelbased approach the typical way to obtain these estimates, by the wave buoy analogy, is to conduct spectral analysis on the measured vessel responses whereafter the obtained response spectra are compared to theoretically calculated ones obtained by combined use of RAOs and a guessed wave spectrum; but iteratively improving the guess by some mathematical technique. The consequence of this approach is that wave amplitude and phase are not directly estimated, since the solution is given in terms of wave spectral ordinates in the frequency domain. The necessity of spectral analysis and associated transformation to frequency domain by standard FFT, or parametric methods [31], [32], implies that the wave estimations are backdated and may be unreliable in case of nonstationary conditions, as discussed previously in Section II. These disadvantages are ever present, to smaller or larger degree, and efforts should/could be introduced to mitigate them; for instance, spectral procedures to handle nonstationary conditions could be introduced/developed [22], [33], [34].

Instead of a solution derived by use of spectral analysis, it has recently been investigated [27] to possibly make the fitting of the measured response and the corresponding theoretically calculated one directly in the time domain. This is done using nonlinear least squares (NLLS) fitting of a batch of time-series data of the response, and hence one advantage is considered to be the fact that calculations of response spectra are unnecessary. It is noteworthy that the use of recursive NLLS methods might be able to provide real-time estimates without the need of using batch data, although this is outside the scope of the current paper.

In the following the proposed solution is discussed and for matters of convenience a specific response, the heave motion $z$, is considered. The solution process is illustrated as a block diagram in Figure 1. The Aranovskiy filter is used to provide the frequency estimate in order to simplify the nonlinear fitting since global convergence otherwise has been found unreliable due to both local minima in the nonlinear cost function and regions with small gradients. Herein, the nonlinear optimisation is implemented with the Levenberg-Marquardt algorithm which is an iterative least squares algorithm addressed to nonlinear minimisation problems specifically. The actual fitting is done using a batch process as shown in Figure 2, where each batch contains measurements from 512 samples equivalent to 51.2 seconds. A batch overlap of $75 \%$ has been used and an estimate is thus calculated every $\frac{51.2}{4}=12.8$ seconds.

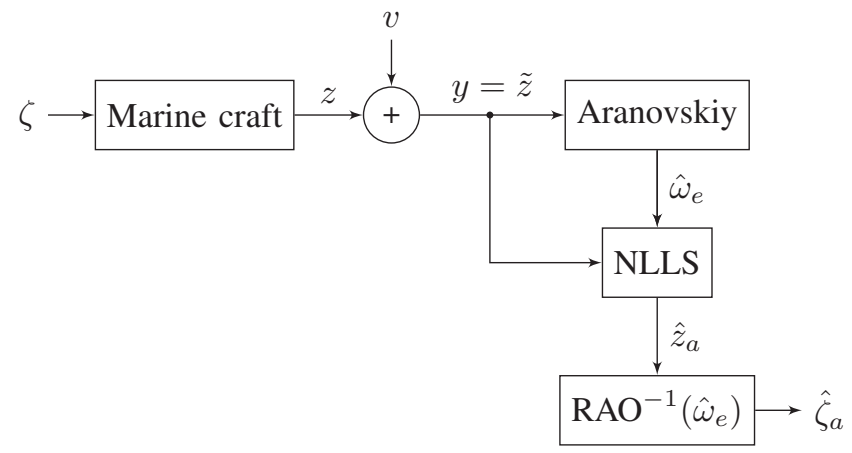

Figure 1: Wave amplitude estimation using nonlinear least squares fitting (NLLS). [27] 


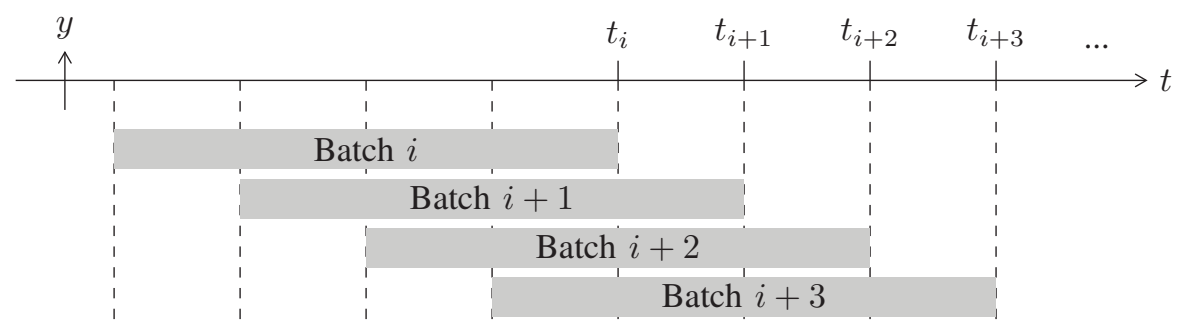

Figure 2: Batch data with $75 \%$ overlap. Batch $i$ is processed at time $t_{i}$. [27]

Obviously, this practice leaves room for a bit of 'tuning' depending on the physical problem; however, in this work such a parameter study has not been considered.

The batch data is fitted with the regression function

$$
y=\hat{z}_{a} \cos \left(\hat{\omega}_{e} t+\hat{\varepsilon}\right)
$$

where the independent variables to be fitted are the heave amplitude estimate $\hat{z}_{a}$ and phase estimate $\hat{\varepsilon}$, respectively. In order to avoid erroneous fitting results, it has been found necessary to split the fitting into two subsequent steps: First fitting the phase $\hat{\varepsilon}$ using a fixed initial amplitude guess $\hat{z}_{a}=\max (y)$ and then fitting the amplitude $\hat{z}_{a}$ using the previously determined phase estimate. This strategy requires the algorithm to be followed twice, thus increasing the computing time. On the other hand, by experimenting with varying initial conditions, calculations have been found more robust against local minima if the following trigonometric relation is used

$$
\begin{aligned}
y & =\hat{z}_{a} \cos \left(\hat{\omega}_{e} t+\hat{\varepsilon}\right) \\
& =\hat{z}_{a} \cos (\hat{\varepsilon}) \cos \left(\hat{\omega}_{e} t\right)-z_{a} \sin (\hat{\varepsilon}) \sin \left(\hat{\omega}_{e} t\right) \\
& =a_{1} \cos \left(\hat{\omega}_{e} t\right)-a_{2} \sin \left(\hat{\omega}_{e} t\right)
\end{aligned}
$$

and fit for both $a_{1}$ and $a_{2}$ simultaneously where $a_{1}=\hat{z}_{a} \cos (\hat{\varepsilon})$ and $a_{2}=\hat{z}_{a} \sin (\hat{\varepsilon})$. The heave amplitude estimate $\hat{z}_{a}$ and phase estimate $\hat{\varepsilon}$, respectively, are thus given by

$$
\begin{aligned}
\hat{z}_{a} & =\sqrt{a_{1}^{2}+a_{2}^{2}} \\
\hat{\varepsilon} & =\operatorname{atan} 2\left(a_{2}, a_{1}\right) ; \quad-\pi<\operatorname{atan} 2(\ldots) \leq \pi
\end{aligned}
$$

Consequently, using Eq. (13) as the regression function, it is only needed to run the NLLS algorithm once.

\section{C.2 Other methods:}

In the original study [27], partly summarised in the present paper, two other procedures were also investigated for their possible usefulness in shipboard sea state estimation. The two methods are based on the Extended Kalman Filter (EKF) [35], [36] and recursive least squares (RLS) fitting, respectively. In any data analysis, the given method would, like NLLS fitting, be a complement to the frequency-estimate by the Aranovskiy filter. In the following, both procedures are briefly outlined, but leaving out mathematical details which can be found in [27].

Independently, EKF or RLS, the frequency-estimate $\hat{\omega}_{e}$ is used to initiate the subsequent estimation process of wave amplitude and phase. If the EKF procedure is applied it is necessary to write the physical model, i.e. the equation of motion, in a state-space representation. This is achieved by modelling the harmonic wave as an undamped oscillator, and the system equations are thus defined by:

$$
\begin{aligned}
\ddot{z}+B\left(\kappa, \omega_{n}\right) \dot{z}+C\left(\omega_{n}\right) z & =F\left(\omega_{e}\right) \zeta \\
\ddot{\zeta} & =-\omega_{e}^{2} \zeta \\
y & =\tilde{z}=z+v
\end{aligned}
$$

where, in general, the motion component $z$ will be a function of the damping ratio $\kappa$, the natural frequency $\omega_{n}$ of the system, and a forcing function $F\left(\omega_{e}\right)$ depending explicitly on the wave (encounter) frequency. Implicitly, the functions $B(\ldots)$, $C(\ldots), F(\ldots)$, depend also on vessel geometry and wave-fluid characteristics. In the lower equation, $v$ is an added noise contribution. In this formulation, the state vector is chosen as $x=\left[\begin{array}{llll}z & \dot{z} & \zeta & \dot{\zeta}\end{array}\right]^{T}$ and the problem is then cast in a matrix form suitable for the EKF, e.g., [35], [36], where the amount of necessary algebraic and mathematical operations depend on the specific physical problem. In the end, the solution leaves a tracking of the wave elevation, represented by the state variable $x_{3}(t)$ that depends on time $t$. Further details are given in [27].

The third approach resembles to some extent the "traditional" approaches of the wave buoy analogy, and the procedure relies on a fitting of response spectra. Specifically, the fast Fourier transform (FFT) of a batch of data samples provides the spectrum, $S_{R}(\omega)$, of the given response $R$. The Aranovskiy

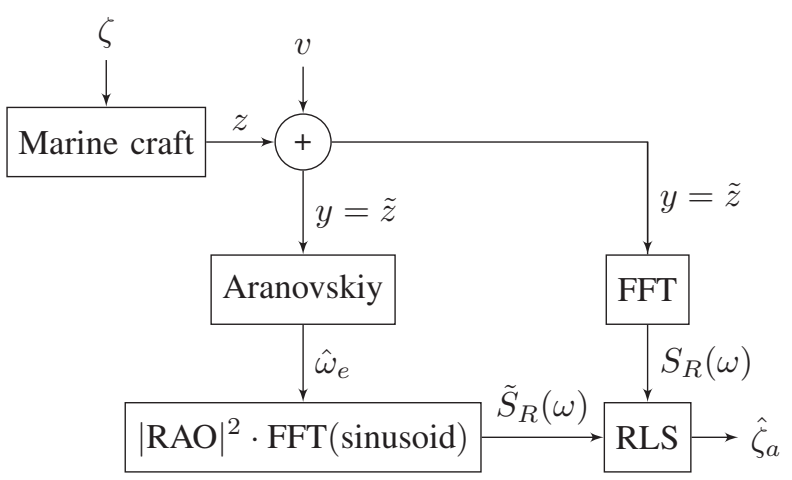

Figure 3: Wave amplitude estimation using RLS fitting of spectrum. 
frequency-estimate and the response RAO, in combination, are used to generate a reference spectrum, $\tilde{S}_{R}(\omega)$, of the response caused by a regular wave with amplitude $\zeta_{a}=1 \mathrm{~m}$. The two spectra are fitted using RLS giving an estimate of the wave amplitude. The procedure is summarised in Figure 3 as a block diagram. In the particular application, the RLS fitting is based on an adaptive algorithm that recursively estimates the wave height by minimising a weighted cost function derived from the response spectra; details and a more general description of RLS fitting are given in [27]. It should be noted that, contrary to the other two methods, the RLS fitting procedure does not yield an estimate of the wave phase which means that the actual wave elevation process cannot be reconstructed fully.

The main focus in the present paper is on the NLLS fitting procedure. Therefore, the case studies considered in the next section are, almost exclusively, dealt with by this procedure in combination with application of the Aranovskiy filter to estimate wave frequency, amplitude and phase. However, a few remarks about the three procedures are put forward in a sort of comparative study made in [27].

\section{EXAMPLES: RESULTS AND DISCUSSIONS}

\section{A. Numerical Simulations at Zero-forward Speed}

Two vessels of different type are studied; a semisubmersible and a container ship. Photos of the container ship and a model-version of the hull structure of the semisubmersible are shown in Figure 4. Main dimensions of the vessels are left out, since the particulars are of little relevance as conceptual examples solely are studied. Independent on vessel type, the considered response is the heave motion, and

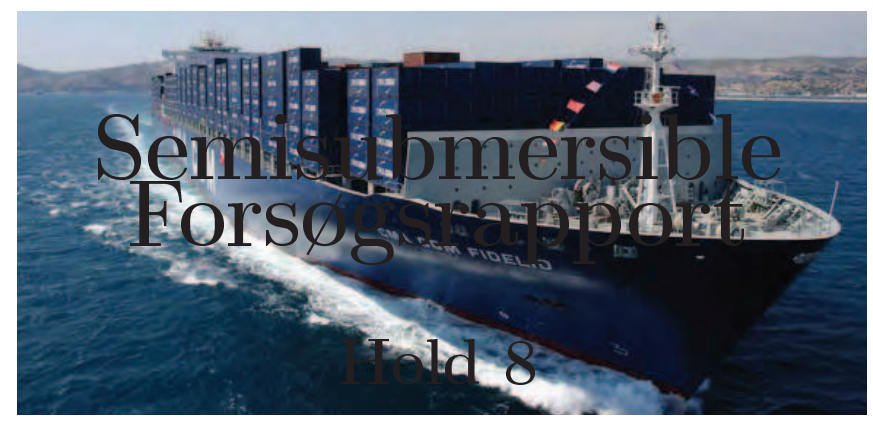

(a) Large panamax container vessel.

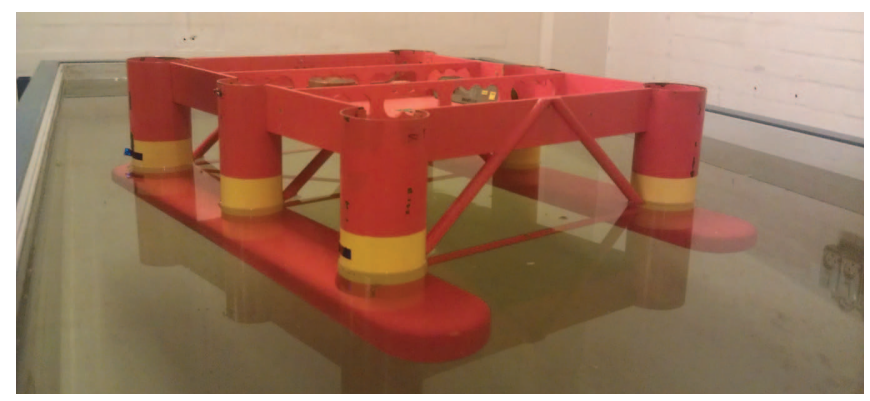

(b) Model-version of the semi-submersible (hull structure only).

Figure 4: Photos of the two considered vessel. in any case the response amplitude operator (RAO) is given in terms of closed-form expressions implemented according to [27] and [30] for the semi-submersible and the container ship, respectively.

The examples deal purely with numerical simulations and apply to long-crested, regular wave trains, numerically simulated in terms of sinusoidal signals. Moreover, the effect of forward speed is not addressed. These somewhat hypothetical conditions are selected because the model-based part, at this stage, is capable to handle only conditions of a seaway made up by a regular wave, although the signal-based part, yielding the encountered peak frequency of a wave system, has been shown previously [25] to provide fair estimates for full-scale operational data of an in-service container ship. Nonetheless, the case studies are limited to regular waves but with results derived for nonstationary conditions.

Altogether, the novel approach for sea state estimation consists of the Aranovskiy filter [28], used to estimate the (encountered) wave peak frequency in a manner similar to [25] and, subsequently, NLLS fitting is applied to estimate wave amplitude and phase. However, before the combined estimation method is illustrated the performance of the Aranovskiy filter is investigated exclusively.

\section{B. Performance of the Aranovskiy Filter}

As reported [27], the behaviour of the filter depends on the chosen cut-off frequency $\omega_{f}$, the observer gain $k_{a}$ and on the measured signal $y$ that carries the information to be estimated. In the following, a general sinusoidal signal with frequency $\omega_{f}=1.0 \mathrm{rad} / \mathrm{s}$ and amplitude $A=A_{y}$ is analysed by the Aranovskiy filter; whether the signal is that of a wave or a response is in this particular case irrelevant.

The effect of the cut-off frequency is shown in [25] where it is concluded that a trade-off is made between convergence rate and steady-state error of the estimate. A low cut-off frequency leads to slower convergence while a larger cut-off frequency results in larger steady-state errors. The choice of observer gain presents a similar trade-off which is seen in Figure 5a. A smaller observer gain results in slower convergence rate while a too large gain increases the steady-state error and introduces oscillations in the estimate. Finally, in Figure $5 \mathrm{~b}$ it seen that the amplitude $A_{y}$ of the measured signal has the same effect on convergence rate and steady-state error.

Simulations with different levels of white Gaussian measurement noise and constant observer gain $k_{a}=0.2$ are shown in Figure 6a. The level of noise is controlled by the signalto-noise ratio SNR. It it seen that high levels of white noise increase the steady-state error and the estimate becomes noisy. The rise-time is not affected significantly. By applying lowpass filtering to the estimated signal, it is possible to obtain a smoother signal with less variance at the expense of a longer rise-time. The filtered estimate is shown in Figure $6 \mathrm{~b}$ where a first order lowpass filter with the time-constant $\tau=30 \mathrm{~s}$ has been applied. The standard deviations are listed in Table I based on 2000 seconds of time-series data after steady-state has been reached. It is seen that the standard deviation is 
Table I: Standard deviation of the estimate for varying levels of measurement noise. [27]

\begin{tabular}{lc|c} 
& & Lowpass filtered \\
\hline SNR & Std. deviation & Std. deviation \\
\hline $0 \mathrm{~dB}$ & $0.0257 \mathrm{rad} / \mathrm{s}$ & $0.0068 \mathrm{rad} / \mathrm{s}$ \\
$6 \mathrm{~dB}$ & $0.0107 \mathrm{rad} / \mathrm{s}$ & $0.0030 \mathrm{rad} / \mathrm{s}$ \\
$12 \mathrm{~dB}$ & $0.0049 \mathrm{rad} / \mathrm{s}$ & $0.0011 \mathrm{rad} / \mathrm{s}$ \\
$18 \mathrm{~dB}$ & $0.0024 \mathrm{rad} / \mathrm{s}$ & $0.0005 \mathrm{rad} / \mathrm{s}$ \\
$24 \mathrm{~dB}$ & $0.0012 \mathrm{rad} / \mathrm{s}$ & $0.0004 \mathrm{rad} / \mathrm{s}$ \\
\hline
\end{tabular}

significantly reduced for the lowpass filtered cases. Moreover, the steady-state errors are seen to become negligible around $12 \mathrm{~dB}$ SNR, corresponding to a signal with four times the power of the noise.

As another test on the performance of the filter, a nonstationary situation is studied where a sudden relatively large change in the frequency of the sinusoidal signal occurs. Basically, this case corresponds to the situation of an advancing vessel that changes its speed (momentarily) resulting in a change in the

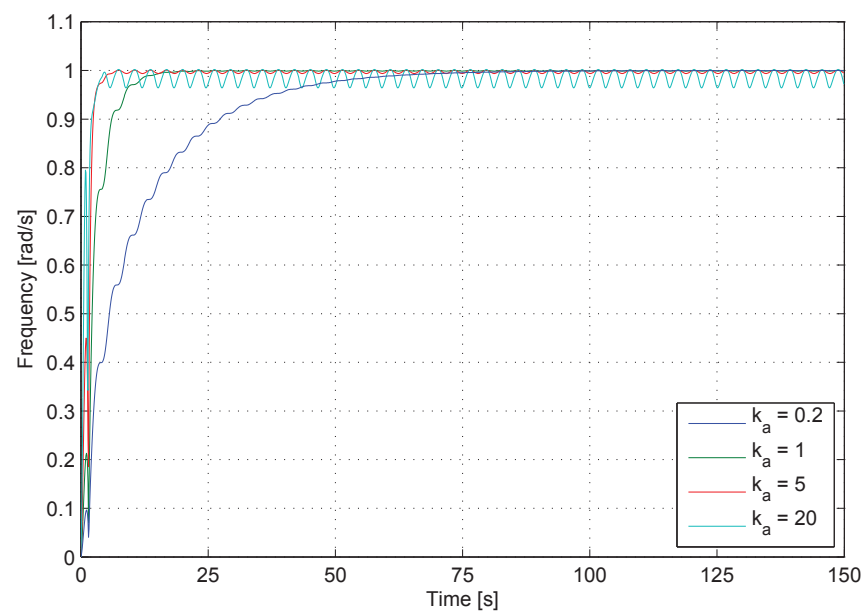

(a) Effect of varying observer gain with $A=1$. [27]

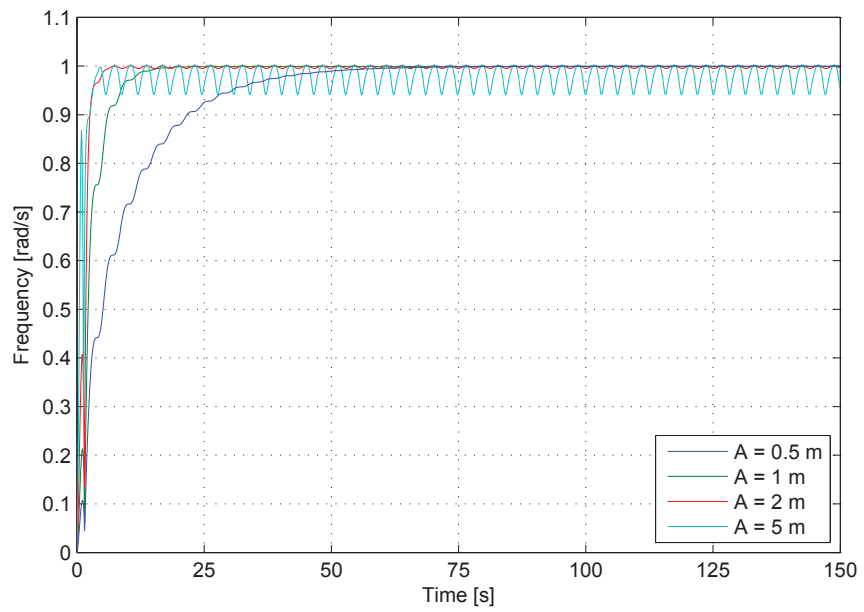

(b) Effect of varying signal amplitude with $k_{a}=1$. [27]

Figure 5: Trade-off between convergence rate and steady-state error. encountered wave frequency. The example is therefore made up by simulating the heave motion of the semi-submersible exposed to a regular wave with changing wave frequency from 0.4 to $1.0 \mathrm{rad} / \mathrm{s}$ at time $t=100 \mathrm{~s}$; although the semi-submersible is at zero-forward speed during the whole process, the sudden change in wave frequency resembles the aforementioned speed change. The result is shown in Figure 7 and it should be noted that measurement noise is not included for this case. From the upper plot it is observed that the sudden change in true frequency introduces transients in the frequency estimate, which shows large oscillations from the time the change occurs but gradually converging to the true value and reaching it after about 200 seconds. The explanation for the misbehaviour of the filter in this intermediate period can be found in the spectrogram of the heave motion. The spectrogram is seen as the lower plot of Figure 7 and it is evident that the transient excites the semisubmersible at the heave resonance frequency $\omega_{n}$. The power contents at the resonance frequency and at the wave frequency,

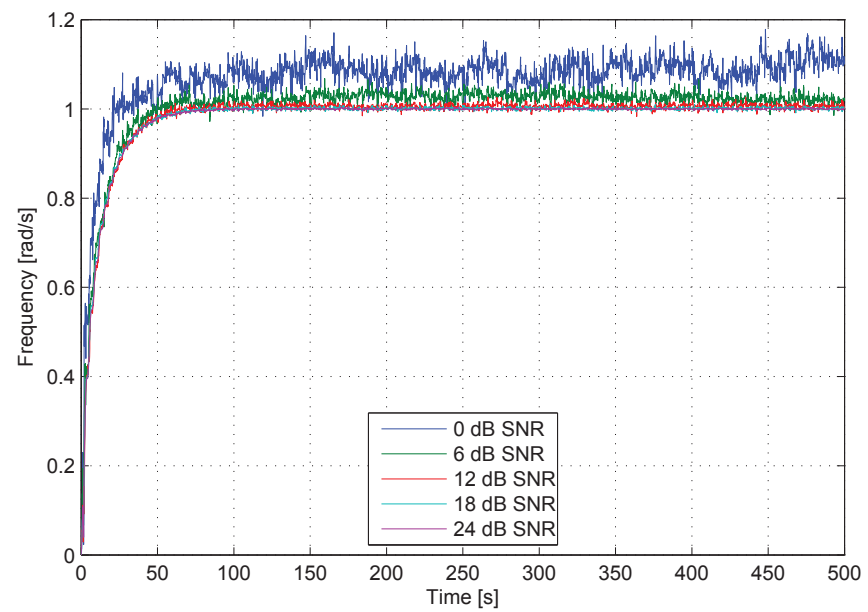

(a) Estimation of sinusoid with Gaussian measurement noise. [27]

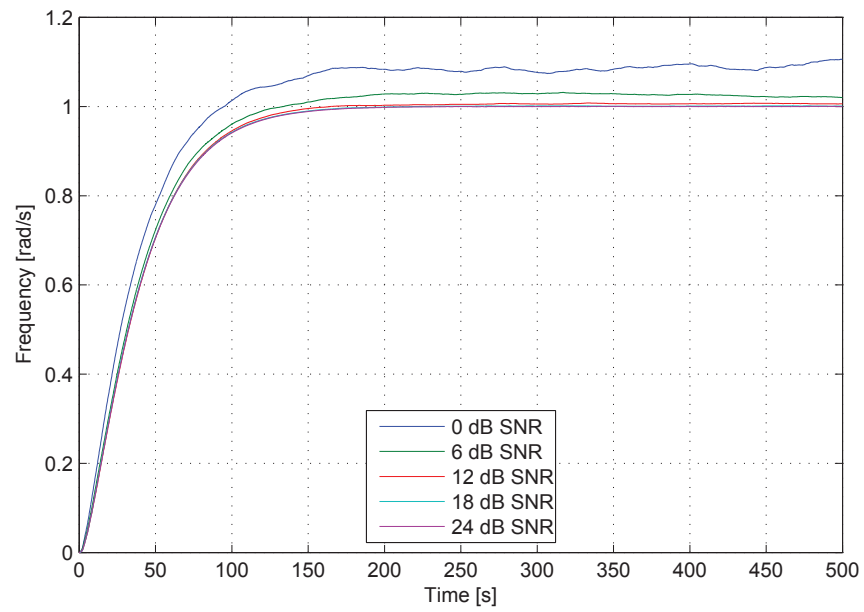

(b) Frequency estimate with lowpass filtering. [27]

Figure 6: Frequency estimation of noisy sinusoid using the Aranovskiy filter. 

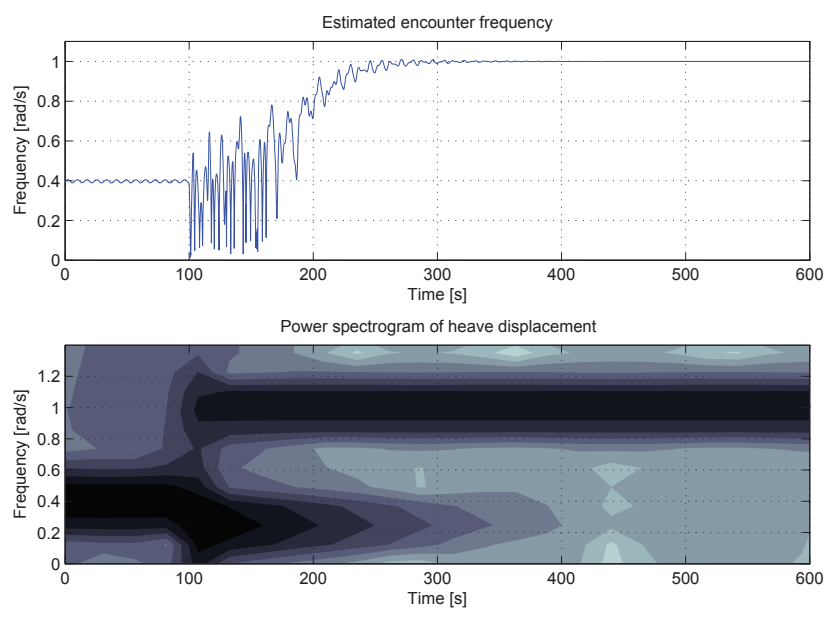

Figure 7: Top: Estimate of "encounter" frequency for a regular wave with changing frequency. [27] Bottom: The associated power spectrogram of the heave position $z$. [27]

respectively, are of similar strength leaving no distinct peak for the Aranovskiy filter to detect. Since the resonance oscillations are only excited at the transient, they will diminish due to the damping in the system. The content at the encounter frequency thus becomes more prominent and the filter converges to the "encounter" frequency with (nearly) no error at steady-state.

In conclusion, it has thus been shown that the Aranovskiy filter is robust to significant levels of measurement noise; although a less elaborate implementation is made in the study herein compared to [25]. Furthermore, the Aranovskiy filter is able to estimate the (encounter) frequency in regular waves during nonstationary conditions.

\section{Analysis of Simulated Vessel Responses}

C.1 Semi-submersible without measurement noise:

In the first case study, the semi-submersible is exposed to regular beam waves in terms of a sinusoidal wave with frequency $\omega=0.6 \mathrm{rad} / \mathrm{s}$, phase $\varepsilon=\pi \mathrm{rad}$, and an initial amplitude $\zeta_{a}=1.0 \mathrm{~m}$ but increasing linearly to $\zeta_{a}=1.5 \mathrm{~m}$ during the time $t=[200 ; 220]$ s. Thus, the measured heave response is simulated by combined use of the wave elevation process and the heave RAO; whereupon the resulting signal is analysed by the wave estimation procedure outlined in Section III and summarised in Figure 1. In this case, measurement noise has not been added and, hence, all parameters are known exactly.

The outcome of the NLLS algorithm is shown in Figure 8 which has the frequency estimate and the amplitude estimate as the upper and the lower plot, respectively. It is observed that that the wave amplitude estimate has converged at about $270 \mathrm{~s}$, which means that the convergence time is near the batch time length (51.2 s); noting that the period of increasing amplitude ends at $220 \mathrm{~s}$. The steady-state error of the amplitude estimate is seen to be insignificant both before and after the increase in wave amplitude. Similarly, the frequency estimate is (close to)
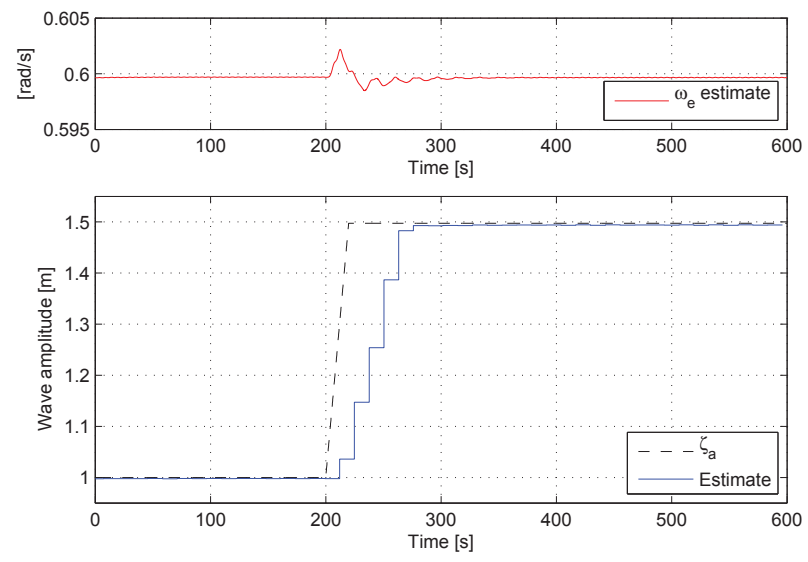

Figure 8: Frequency estimate (top) by Aranovskiy filter and wave amplitude estimate (bottom) using NLLS fitting. [27]

spot on before and after the increase; and with a very small relative error during the period where the increase in wave amplitude occurs.

The estimated wave phase is shown in Figure 9, and it is observed that the estimate is rather sensitive to the wave frequency estimate. This is explained by looking at the instantaneous phase of the wave given by $\hat{\omega}_{e} t+\hat{\varepsilon}$. Any uncertainty in the first term will be compensated with the phase estimate $\hat{\varepsilon}$ during the NLLS fitting process. As time $t$ increases, any uncertainty in $\hat{\omega}_{e}$ increases proportionally. The estimation procedure therefore needs to change the phase estimate $\hat{\varepsilon}$ in order to minimise the cost function. It is however still possible to reconstruct the wave elevation using the estimated wave frequency $\hat{\omega}_{e}$, wave amplitude $\hat{\zeta}_{a}$ and phase delay $\hat{\varepsilon}$, since the estimated instantaneous phase error is minimised. Indeed, this is seen by Figure 10 where the complete wave elevation process is shown as the top plot, containing both the estimated and the true process. The bottom plot depicts the reconstruction error, and it is evident that the agreement is
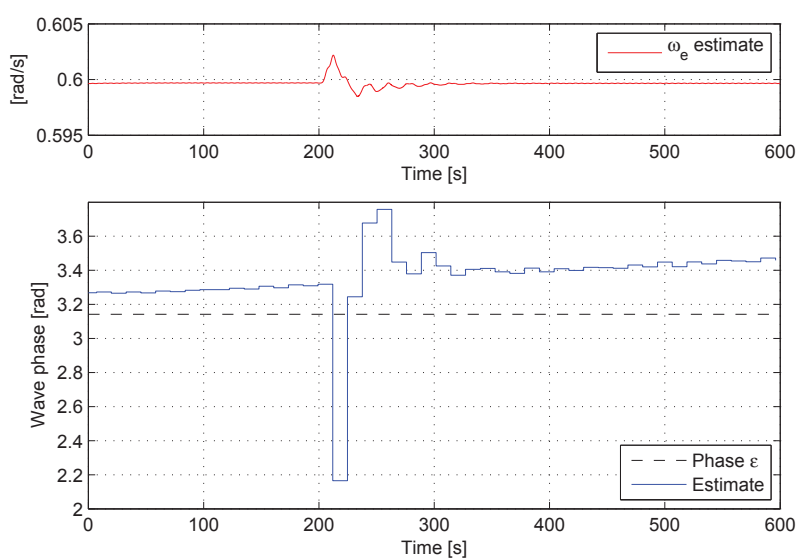

Figure 9: Phase estimation (bottom) together with frequency estimate (top). [27] 

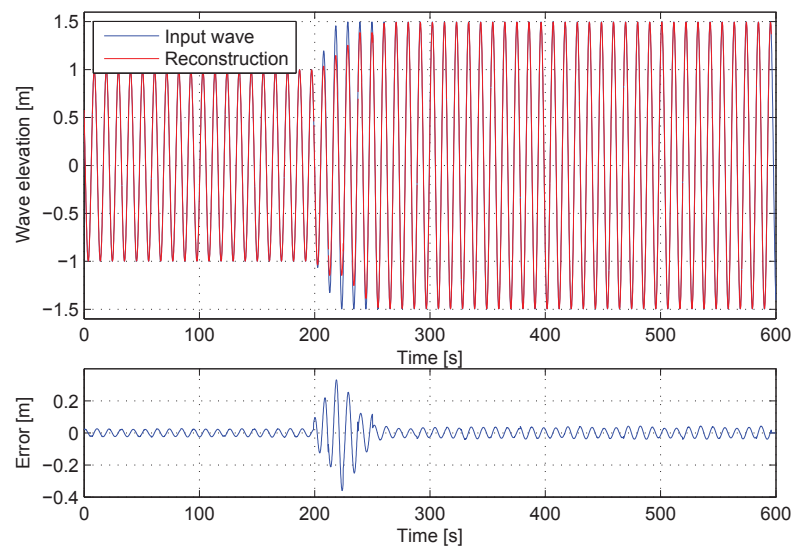

Figure 10: Top: Reconstruction of wave elevation based on estimated parameters. Bottom: Reconstruction error. [27]

good. The actual reconstruction of the wave elevation process is made by combining segments from each of the batch estimates. As illustrated by Figure 2, the estimate from batch $i$ is used for the reconstruction in the time interval $\left[t_{i-1} ; t_{i}\right]$. At steady-state the reconstruction error shows oscillations with an amplitude less than $3 \%$ of the wave amplitude.

C.2 Container ship with measurement noise:

This case study is made for the container ship being exposed to a regular wave train; nearly identical to that of the previous

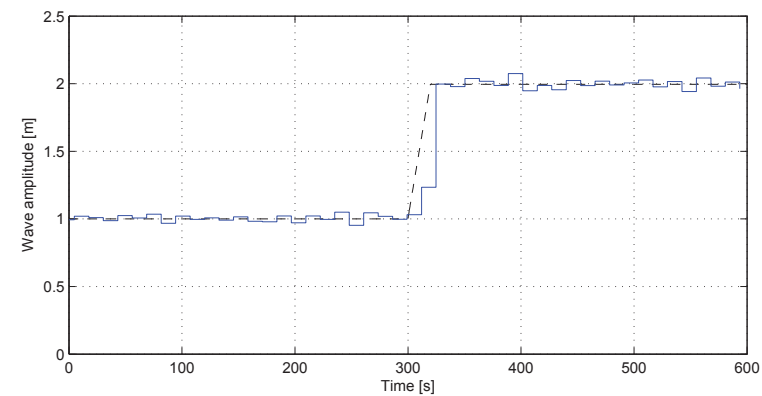

(a) Estimated wave amplitude

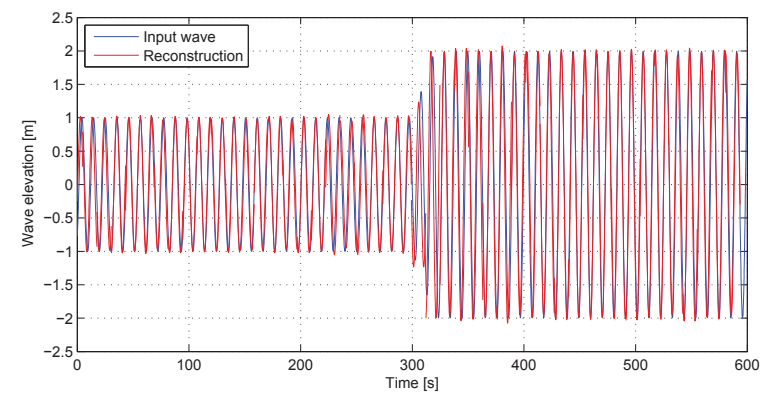

(b) Reconstruction of wave elevation process.

Figure 11: Sea state estimation based on heave response of the container vessel exposed to bow-quartering regular waves $(U$ $=0$ knots) and considering measurement noise (12 dB SNR). case study: $\omega=0.6 \mathrm{rad} / \mathrm{s}, \varepsilon=\pi \mathrm{rad}, \zeta_{a}=1.0 \mathrm{~m} \rightarrow 2.0 \mathrm{~m}$ during the time $t=[300 ; 320]$ s. The main difference in the present case study is the addition of measurement noise taken as Gaussian white noise produced with a $12 \mathrm{~dB}$ SNR and, moreover, the measured heave response is simulated in a seaway with bow-quartering waves (relative wave heading equal to $135 \mathrm{deg}$ ).

The wave amplitude estimate, from a single simulation, is shown in Figure 11a and the complete reconstruction of the wave elevation process in Figure 11b. The plots show that the wave parameters, including the actual time history, are estimated with a reasonable accuracy. The accuracy can be quantified by running multiple simulations and based on findings, equivalent to those illustrated by Figure 11, it is concluded that the reconstruction error is less than $\pm 15 \%$ of the wave amplitude in case of measurement noise with $12 \mathrm{~dB}$ SNR. It is noteworthy that similar analyses have been made for the semi-submersible [27], where the findings were the following: a reconstruction error less than $\pm 30 \%$ of the wave amplitude for $9 \mathrm{~dB}$ SNR, $\pm 20 \%$ for $12 \mathrm{~dB}$ SNR and $\pm 15 \%$ for $18 \mathrm{~dB}$ SNR, respectively.

\section{Other Methods: EKF and RLS}

The two other methods proposed for sea state estimation were based on the Extended Kalman Filter (EKF) and recursive least squares (RLS) fitting. A large discussion on their application to data is beyond the scope of this paper but many findings can be found in [27], which examines results/estimates using simulated measurements of the semisubmersible's heave response (with measurement noise). A comparison in this respect, can be seen from Figure 12, where the three procedures - NLLS, EKF, and RLS - are compared for different levels of measurement noise. It can be observed that the EKF method is more precise, both in terms of steadystate error and variance. The RLS and NLLS methods show a slightly reduced performance in this regard; anyhow, while not as precise as the EKF method, the estimated steady-state error is less than $3 \%$ of the true wave height, based on noisy measurements with $9 \mathrm{~dB}$ SNR.
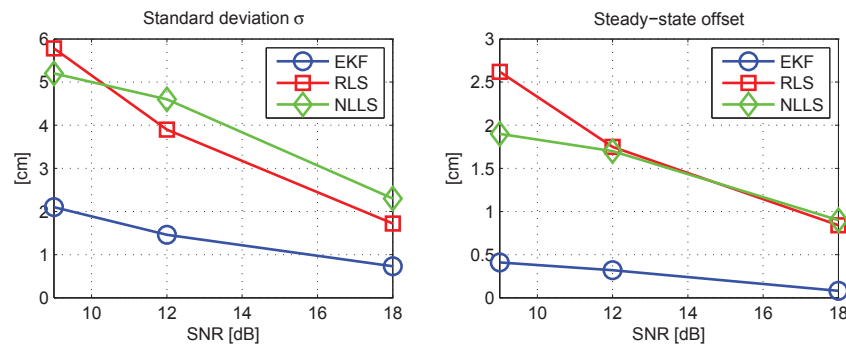

Figure 12: Comparison of amplitude estimates. [27]

\section{CONCLUDING REMARKS}

Methods for shipboard estimation of the descriptive parameters of regular waves have been developed and tested. Special attention was given to a combined procedure based 
on the Aranovskiy filter [28] and subsequent nonlinear least squares (NLLS) fitting; used to estimate the (encountered) wave peak frequency and, respectively, applied to estimate wave amplitude and phase. It was demonstrated that the procedure provides accurate estimates for two different types of vessels; both exposed to regular wave trains and at zeroforward speed.

One main advantage of the method's first part, i.e. the estimate of the wave frequency by the Aranovskiy filter, is that this part is purely signal-based and thus will be unaffected by (uncertain) model parameters. The second part of the procedure, estimating amplitude and phase, is model-based for what reason availability of accurate transfer functions to model the particular vessel's hydrodynamic behaviour is fundamental. Ultimately, it would be of much interest to make the combined method fully signal-based but, indeed, this will require some elaborate and novel approaches and, currently, their development are difficult to predict about. However, some other elaborations and suggested further developments could be seen as important and useful. This would include, but not necessarily limited to nor in the arranged order:

- Verification of the presented results using model-scale experiments with regular waves.

- The application of the Aranovskiy filter requires the gain to be tuned properly, and efforts could be made to allow the gain tuning to be completely automated, which is not the case in the procedure as is. Work in this direction has been explored already and one feasible approach is developed in [25].

- Until now, cases of zero-forward speed have been considered only and, obviously, the procedure should also be capable to handle data from advancing marine crafts.

- As an intermediate stage, the extension to consider a regular (sinusoidal) wave train composed by two wave components could be beneficial, as it would provide knowledge about how to handle estimation of an irregular wave train made up by a (very) large number of regular wave components. Specifically, work could address the use of several notch or bandpass filters to select individual harmonic components from a wave spectrum, and then use regular wave estimators in parallel for each component. In the end, this would make the method applicable to real (full-scale) data.

- The combination/consideration of several responses simultaneously, e.g., \{heave; roll; pitch\} could possibly be used to estimate also the relative wave heading.

\section{ACKNOWLEDGMENT}

The work of the first author was partly supported by the Research Council of Norway through the Centres of Excellence funding scheme, Project number 223254-AMOS.

\section{REFERENCES}

[1] M. Huss and A. Olander, "Theoretical Seakeeping Predictions On Board Ships - A System for Operational Guidance and Real Time Surveillance," Naval Architecture, Department of Vehicle Engineering, Royal Institute of Technology, Tech. Rep., 1994.
[2] H. Chen, V. Cardone, and P. Lacey, "Use of Operation Support Information Technology to Increase Ship Safety and Efficiency," Trans. of SNAME, vol. 106, pp. 105-127, 1998.

[3] H. Payer and H. Rathje, "Shipboard Routing Assistance Decision Making Support for Operation of Container Ships in Heavy Seas," Trans. of SNAME, vol. 112, pp. 1-12, 2004.

[4] E. Bitner-Gregersen and R. Skjong, "Concept for a risk based Navigation Decision Assistant," Marine Structures, vol. 22, pp. 275-286, 2009.

[5] U. D. Nielsen, P. Friis-Hansen, and J. J. Jensen, "A Step towards riskbased decision support for ships - Evaluation of limit states using parallel system analysis," Marine Structures, vol. 22, pp. 209-224, 2009.

[6] U. D. Nielsen, Z. Lajic, and J. J. Jensen, "Towards fault-tolerant decision support systems for ship operator guidance," Reliability Engineering and System Safety, vol. 104, pp. 1-14, 2012.

[7] U. D. Nielsen and T. Iseki, "Prediction of First-Order Vessel Responses with Applications to Decision Support Systems," in Proc. of 5th WMTC (to appear), Providence, RI, USA, 2015.

[8] S. V. Hansen, "Performance Monitoring of Ships," Ph.D. dissertation, Department of Mechanical Engineering, Technical University of Denmark, 2012.

[9] B. P. Pedersen, "Data-driven Vessel Performance Monitoring of Ships," Ph.D. dissertation, Department of Mechanical Engineering, Technical University of Denmark, 2015.

[10] A. Brodtkorb, A. J. Sørensen, and A. R. Teel, "Increasing the Operation Window for Dynamic Positioned Vessels Using the Concept of Hybrid Control," in Proc. of 33rd OMAE, San Francisco, CA, USA, 2014.

[11] A. Brodtkorb, A. R. Teel, and A. J. Sørensen, "Sensor-Based Hybrid Observer for Dynamically Positioned Vessels," in Proc. of 54th IEEE Conference on Decision and Control (to appear in), Osaka, Japan, 2015.

[12] J. Hua and M. Palmquist, "Wave Estimation through Ship Motion Measurement," Naval Architecture, Department of Vehicle Engineering, Royal Institute of Technology, Tech. Rep., 1994.

[13] T. Iseki and K. Ohtsu, "Bayesian estimation of directional wave spectra based on ship motions," Control Engineering Practice, vol. 8, pp. 215 $219,2000$.

[14] E. A. Tannuri, J. V. Sparano, A. N. Simos, and J. J. D. Cruz, "Estimating directional wave spectrum based on stationary ship motion measurements," Applied Ocean Research, vol. 25, pp. 243-261, 2003.

[15] U. D. Nielsen, "Estimations of on-site directional wave spectra from measured ship responses," Marine Structures, vol. 19, pp. 33-69, 2006.

[16] R. Pascoal and C. G. Soares, "Kalman filtering of vessel motions for ocean wave directional spectrum estimation," Ocean Engineering, vol. 36, pp. 477-488, 2009.

[17] N. Montazeri, U. D. Nielsen, and J. J. Jensen, "Estimation of wind sea and swell using shipboard measurements - A refined parametric modelling approach," Applied Ocean Research, 2015 (under review).

[18] U. D. Nielsen and D. C. Stredulinksy, "Sea state estimation from an advancing ship - A comparative study using sea trial data," Applied Ocean Research, vol. 34, pp. 33-44, 2012.

[19] U. D. Nielsen, I. M. V. Andersen, and J. Koning, "Comparisons of Means for Estimating Sea States from an Advancing Large Container Ship," in Proc. 12th PRADS, Changwon, South Korea, 2013.

[20] A. N. Simos, J. V. Sparano, E. A. Tannuri, and V. L. F. Matos, "Directional Wave Spectrum Estimation Based on a Vessel 1st Order Motions: Field Results," in Proc. of 17th International Offshore and Polar Engineering Conference, Lisbon, Portugal, 2007.

[21] J. V. Sparano, E. A. Tannuri, A. N. Simos, and V. L. F. Matos, "On the Estimation of Directional Wave Spectrum Based on Stationary vessels 1st Order Motions: A New Set of Experimental Results," in Proc. of OMAE'08, Lisbon, Portugal, 2008.

[22] C. Møgster, "Bayesian methods for estimating non-stationary ship response spectra," Master's thesis, Norwegian University of Science and Technology, Trondheim, Norway, 2015.

[23] T. Iseki and U. D. Nielsen, "Study on a Short-term Variability of Ship Responses in Waves," Journal of Japan Institute of Navigation, vol. 132, 2015.

[24] D. J. W. Belleter, D. A. Breu, T. I. Fossen, and H. Nijmeijer, "A Globally K-Exponentially Stable Nonlinear Observer for the Wave Encounter Frequency," in Proc. of 9th IFAC Conf. CAMS, Osaka, Japan, 2013.

[25] D. J. W. Belleter, R. Galeazzi, and T. I. Fossen, "Experimental verification of a global exponential stable nonlinear wave encounter frequency estimator," Ocean Engineering, vol. 97, pp. 48-56, 2015. 
[26] A. Brodtkorb, U. D. Nielsen, and A. J. Sørensen, "Sea State Estimation Using Model-scale DP Measurements," in Proc. of MTS/IEEE OCEANS15 (to appear in), Washington, DC, USA, 2015.

[27] M. Bjerregård, "Methods for sea state estimation," Master's thesis, Technical University of Denmark, Kgs. Lyngby, Denmark, 2014.

[28] S. V. Aranovskiy, A. A. Bobtsov, A. S. Kremlev, and G. V. Lukyanova, "A robust algorithm for identification of the frequency of a sinusoidal signal," Journal of Computer and Systems Sciences International, vol. 46, pp. 371-376, 2007.

[29] P. T. Pedersen, P. Andersen, and C. Aage, Grundlagggende Skibs- og Offshoreteknik. Technical University of Denmark, 2010, (in Danish).

[30] J. J. Jensen, A. E. Mansour, and A. S. Olsen, "Estimation of ship motions using closed-form expressions," Ocean Engineering, vol. 31, pp. 61-85, 2004.

[31] H. Akaike and T. Nakagawa, Statistical Analysis and Control of Dynamic Systems. KTK Scientific Publishers, 1988.

[32] A. Neumaier and T. Schneider, "Estimation of Parameters and Eigenmodes of Multivariate Autoregressive Models," ACM Transactions on Mathematical Software, vol. 27, pp. 27-57, 2001.

[33] X. Jiang and G. Kitagawa, "A Time Varying Coefficient Vector AR Modeling of Nonstationary Covariance Time Series," Signal Processing, vol. 33, no. 3, pp. 315-331, 1993.

[34] T. Iseki and D. Terada, "Study on Real-time Estimation of the Ship Motion Cross Spectra," Journal of Marine Science and Technology, vol. 7, pp. 157-163, 2002.

[35] R. G. Brown and P. Y. C. Hwang, Introduction to Random Signals and Applied Kalman Filtering, 3rd ed. John Wiley \& Sons, Inc., 1992.

[36] D. Simon, Optimal State Estimation. John Wiley \& Sons, Inc., 2006. 